\title{
Eosinophilic esophagitis: an interdisciplinary clinical problem
}

\author{
Oksana Wojas ${ }^{1}$, Marta Żalikowska-Gardocka², Edyta Krzych-Fałta ${ }^{1}$, Bolesław Samoliński ${ }^{1}$, Adam Przybyłkowski $^{2}$ \\ ${ }^{1}$ Department of Prevention of Environmental Hazards and Allergology, Medical University of Warsaw, Warsaw, Poland \\ ${ }^{2}$ Department of Gastroenterology and General Medicine, Medical University of Warsaw, Warsaw, Poland \\ Adv Dermatol Allergol 2021; XXXVIII (1): 36-42 \\ DOI: https://doi.org/10.5114/ada.2019.87237
}

\begin{abstract}
Eosinophilic esophagitis is a newly described entity of increasing incidence. Previously thought to be a variation of gastroesophageal reflux disease, now it becomes well known among gastroenterologists, allergologists, paediatricians and pathologists. Aetiology of the disease is strongly correlated with atopic and allergic disorders but exact pathogenesis and cellular mechanisms of inflammatory process in the esophagus are still unknown. Diagnostic criteria have been described but diagnostic tools are still in the research and improvement phase. Clinical manifestation varies considerably between age groups, which causes a delay in the course of diagnosis due to improperly recognized symptoms. Since eosinophilic esophagitis is a chronic disease without a tendency to be self-limiting, delayed diagnosis may lead to complications associated with oesophageal tissue remodelling. Some forms of treatment are approved and of great therapeutical value, nevertheless clinical trials of new medications provide new possibilities. Therefore, many questions regarding eosinophilic esophagitis arise and are still unanswered.
\end{abstract}

Key words: eosinophilic esophagitis, endoscopy, allergy, atopy, glucocorticosteroids.

\section{Introduction}

Eosinophilic esophagitis (EoE), a disease of increasing incidence has been a point of interest in many fields of medicine, mainly gastroenterology, allergology, paediatrics and pathology. EoE is a newly identified entity, first reported about 20 years ago. At that time gastroesophageal reflux disease (GERD) was considered to be a cause of eosinophilic infiltrations in the mucosa of the esophagus. Since some patients with oesophageal eosinophilia responded to proton pump inhibitor treatment, a new term, PPI responding oesophageal eosinophilia was introduced. Nevertheless, case studies of patients with EoE with features different to GERD were published. In 1978, Landres et al. reported a case of a patient with achalasia, concomitant hyperplasia of oesophageal muscle membrane and oesophageal eosinophilia [1]. Authors concluded that said patient suffered from a variant of eosinophilic gastritis and enteritis predisposing to disturbances in motor functions of the esophagus. In 1981, Picus and Frank reported a case of a 16-year-old boy with progressive dysphagia and eosinophilic infiltration in the esophagus [2]. Additional tests revealed oesophageal stricture and peripheral blood eosinophilia. Nevertheless, the case was considered to be a variation of eosinophilic gastritis and enteritis. In 1989, Attwood et al. published a work titled: "Esophageal asthma - an episodic dysphagia with eosinophilic infiltrates" [3]. A key point of their work was identification of a group of patients with different severity of dysphagia and normal pH-monitoring. However, 4 years later, the authors reported a new clinical syndrome described as severe eosinophilic infiltration (more than 20 eosinophils in the high power field) in the esophagus of patients with dysphagia and normal pH-monitoring. It was originally named oesophageal eosinophilia with dysphagia, now being known as EoE [4]. One year later, Straumann et al. introduced a new entity called idiopathic EoE [5]. They presented the results of a 4-year observational study performed on a group of 10 patients with recurrent acute dysphagia, discrete changes in endoscopic examination and severe eosinophilic oesophageal infiltrations, in whom improvement was reached after glucocorticosteroids and antihistamines therapy. Therefore, two works, published by two independent authors, coming from different continents, namely Dr Straumann, gastroenterologist, and Dr Attwood, surgeon, laid foundations for further research on classifications, epi-

Address for correspondence: Marta Żalikowska-Gardocka MD, PhD, Department of Gastroenterology and General Medicine, Medical University of Warsaw, 1a Banacha St, 02-097 Warsaw, Poland, phone: +48 2259928 38, fax: +48 225991838 , e-mail: zalikowska@gmail.com, kgw@spcsk.pl Received: 10.06.2019, accepted: 29.06.2019. 
demiology, pathogenesis, clinical picture and treatment of EoE [6]. Originally EoE was considered to be an atopic disease of the esophagus. The role of allergy in its pathogenesis was suggested for the first time in research led by Kelly et al. in 1995, where, in 10 children with vomiting, abdominal pain and eosinophilic oesophageal infiltrations, elemental diet was introduced with improvement [7]. Further research confirmed a significant contribution of allergy. According to up-to-date studies, even $70 \%$ to $90 \%$ of patients with EoE are diagnosed with atopy and allergy, and their role in pathogenesis of EoE is indisputable [8].

\section{Definition}

In accordance with current guidelines, EoE is a chronic inflammatory disease of immunologic aetiology, induced by ingested and inhaled allergens, leading to clinical symptoms, associated with impaired oesophageal function and histopathological predominance of eosinophils in inflammatory infiltrates. It is very important to approach EoE as a clinical-pathological entity, which means the diagnosis of EoE can be reached and the outcomes of treatment monitored only if symptoms of oesophageal function are accompanied by histological changes revealed in oesophageal biopsy $[9,10]$.

\section{Epidemiology}

In recent years the incidence of EoE has been increasing in both children and adults. Morbidity varies from 1 to 20 cases per 100000 per year. Incidence is estimated as 13-49 EoE cases per 100,000 in the general population [9]. In a group of children who underwent gastroscopy, the prevalence rate was 3.7\%, irrespective of the reason for the test. If dysphagia was the indication for gastroscopy, the rate ranged from $63 \%$ to $88 \%$ [9]. Studies in Central Spain in 2005-2011 showed an average annual incidence rate of approx. 6.37/100,000 people, and the prevalence rate was $44.6 / 100,000$ people [11]. The increase in the prevalence rate in recent years may be a result of growing awareness of the disease, better access to endoscopic examinations and improved quality of endoscopic equipment as well as increased incidence of allergies. Eosinophilic esophagitis is diagnosed in people of all races and in all continents, although it is definitely more prevalent in Caucasians [9]. The disease is common in Western countries, particularly in the USA and Europe as well as Australia. It is less common in Asia. The first case of EoE in Japan was reported in 2006. In a Japanese study of 8,589 patients who underwent gastroscopy, Sato et al. found that 17 of the patients had EoE. This group included 13 patients with allergies and most of the EoE subjects were men [12].

\section{Aetiology and pathogenesis}

Eosinophilic esophagitis is more frequent among men than women $(3: 1)$. Adults are usually diagnosed with EoE between the ages of 30 and 50, whereas children between the ages of 5 and 10 [9]. Male gender and Caucasian race are considered to be risk factors for EoE [13]. Allergic diseases, including allergic rhinitis, bronchial asthma and atopic dermatitis along with atopy (defined as increased concentration of IgG antibodies directed against many allergens) are also acknowledged risk factors [13]. Concluding, a phenotype of a typical EoE patient is a young Caucasian man with atopy.

The aetiology of EoE is not clearly defined. Undoubtedly, genetic, immunological and environmental factors take part in the pathogenesis of EoE [9]. Familial occurrence is fairly frequent (10\% of cases) [14]. Many studies put an emphasis on the role of genetic disorders such as Single Nucleotide Polymorphisms (SNPs) in the eotaxin-3 gene on the chromosome 7q11, polymorphism of the transforming growth factor $\beta 1$ (TGF- $\beta 1$ ) gene on the chromosome 19q33, and the polymorphism of the TSLP gene on the chromosome 5q22 [11]. Avila-Castellano et al. reported that a genetic variation in the Tolllike receptor 3 (TLR3) may be a major factor predisposing to EoE [15]. Many epidemiological and clinical studies confirm a strong correlation between EoE and allergic diseases. Eosinophilic esophagitis, bronchial asthma, atopic dermatitis and allergic rhinitis are all diseases of similar features, such as eosinophilic infiltrates, epithelial cell hyperplasia or mucous membrane remodelling [16]. Therefore, EoE is often referred to as oesophageal asthma [13]. Moreover, a correlation between the disease and food allergies is unquestionable [17]. There is plenty of evidence proving the role of Th2-dependant reactions in the pathogenesis of EoE. It is assumed that food and inhalant allergens (swallowed pollens) contact directly with the mucus membrane of the esophagus, where they are presented to T-lymphocytes by dendritic cells. Many proinflammatory cytokines and chemokines are involved in the process $[18,19]$. One of the main cytokines, produced by Th2 and ILC2 is interleukin 5 (IL-5). It is crucial for eosinophils. Apart from inducing their development, it enhances their activity and improves vitality (inhibits the process of apoptosis). Production of IL-5 depends on IL-3, which induces production of eotaxin 3, a chemokine responsible for eosinophil chemotaxis. Eosinophilic granulocytes are not detected in the esophagus of healthy people. The number of eosinophils in blood and tissues is increased in allergic diseases. Chemotactic and activating agents, such as IL-5, granulocyte-macrophage colony-stimulating factor (GM-CSF), IL-2, IL-3, IL-4, IL-16 and tumor necrosis factor $\alpha$ (TNF- $\alpha$ ) as well as eotaxins (CCL11, CCL24, CCL26) are responsible for this phenomenon. It is believed that eosinophils participate, or even predominate, in the destruction and, subsequently, 
remodelling of tissues. This process is a result of a release of substances from eosinophils, namely main basic protein (MBP), eosinophil cationic protein (ECP) and eosinophil peroxidase (EPO) [18]. By releasing the abovementioned proteins from toxic granules, eosinophils take part in forming the defence barrier against allergens and microbes. In case of impaired function of the epithelial barrier, eosinophils participate in defence targeted tissue fibrosis, but simultaneously promote further epithelial damage and perpetual inflammation. TGF- $\beta$ and PDGF also play a part in tissue remodelling, especially in fibrosis processes [20].

\section{Clinical picture}

Symptoms of EoE are a result of esophagus dysfunction and affect patients of different age. The course of the disease may vary. Some patients experience constant symptoms of EoE, often with a tendency to increase, while other patients experience periods of aggravation and remission. The average time of symptoms until diagnosis is made ranges between 3 and 5 years. However, a single acute episode, typically food impaction, may be sufficient for a patient to be diagnosed with EoE [21]. The character and severity of symptoms depend on the patient and may vary in different age groups. The prevalent symptoms reported by pre-school children include stomach ache, vomiting, spitting up and feeding problems, resulting in body weight loss and developmental disorders. These children often fuss and have sleep problems. The

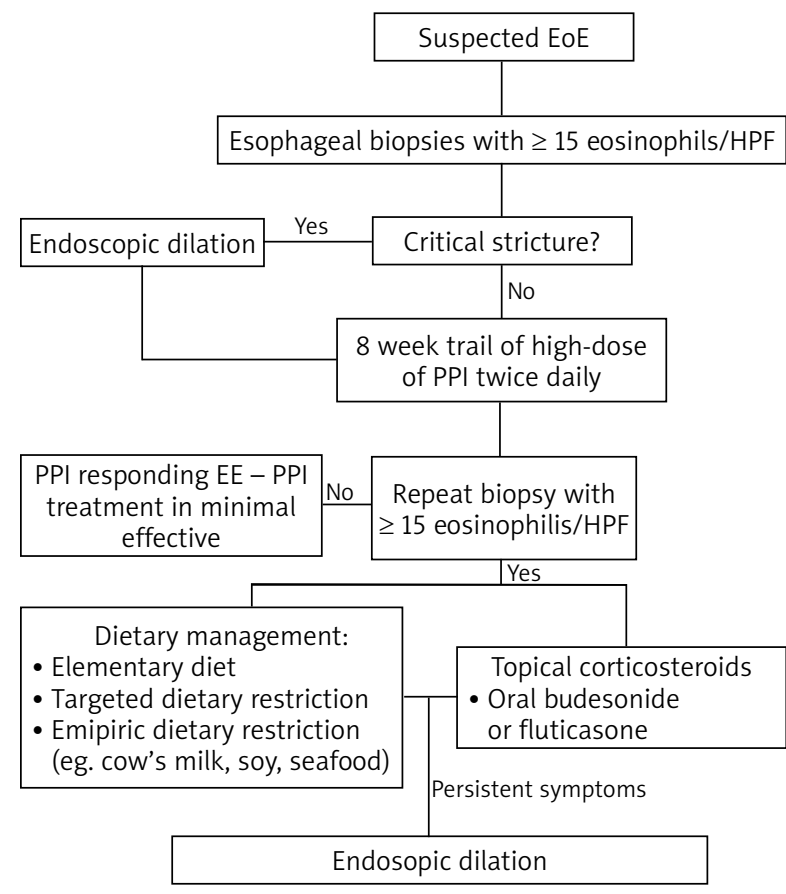

Figure 1. Diagnostic algorithm of eosinophilic esophagitis (EoE) most common symptoms reported by primary school children include aversion to certain foods, preference of liquid diets and mushy foods, a tendency to prolong a meal ("slow eating") as well as stomach ache and vomiting. Atypical symptoms are sometimes reported, e.g. neck pain. Older children and teenagers commonly complain of dysphagia. They tend to prefer mushy foods and to drink while eating and may develop eating-related anxiety disorders. The first symptoms reported by children often include diarrhoea and bloody stools, which makes it more difficult to reach the correct diagnosis. Adults commonly present with dysphagia and food bolus impaction as well as heartburns, belching, retrosternal pain, prolonging a meal and having to drink while eating, changes of eating habits. Unspecific throat pain may often be the only manifestation of EoE in adults, being the cause of laryngological diagnostic tests. The differences in the clinical picture of EoE between age groups are linked with severity of the inflammation process, its duration and the fact that children find it far more difficult to describe their symptoms [9, 20]. Gomez Torrijos et al. advise doctors to ask the following questions when taking medical history: "Do you avoid eating certain foods such as bread, rice, or meat?", "Are you the last to get up from the table?", "Do you drink plenty of water during the meal to help you swallow?" and "Do you cut the food into very small pieces?" [11]. These simple questions can be very helpful in the diagnostic process. The authors emphasise that some patients can develop adaptation habits masking the disease. That can be manifested in "intuitive" diet changes in order to alleviate the symptoms. In conclusion, signs of EoE do not fully correlate with severity of oesophageal inflammation and cannot be the only criterion for assessing effectiveness of the treatment $[9,11]$.

\section{Natural history of the disease}

Eosinophilic esophagitis is a chronic disease without a tendency to be self-limiting. Observations show that the main complication of untreated EoE is oesophageal strictures, which lead to malnutrition. Complications, such as metaplasia or tumours, have not been reported. It seems, however, that patients with EoE require careful long-time observation focused on oesophageal epithelium [11].

\section{Diagnosis}

A double-track approach involving gastroenterology and allergology should be taken when diagnosing EoE (Figure 1).

The following criteria must be met to diagnose the disease:

- clinical symptoms of oesophageal dysfunction, 
- an oesophageal biopsy showing inflammation with at least 15 eosinophils per high-power field with 400x magnification, and

- eosinophilic inflammation limited to the esophagus [9, $11,13,20]$.

The main examination for any patient with symptoms of EoE is esophagogastroduodenoscopy. Endoscopic findings may vary, showing white effusions and exudates, oesophageal rings and uneven lumen of the esophagus (feline esophagus), furrows (esophageal trachealization), strictures, erosions, oedema and mucosal erythema as well as normal appearance of the esophagus $[11,13,20]$ (Figures 2, 3). The endoscopic picture of the esophagus is normal in $10 \%$ of adults and $32 \%$ of children with EoE [11]. It is important to note that lesions revealed in endoscopy are not pathognomonic for this disease. Therefore, a biopsy is required in each case [11]. Taking a total of six samples from the distal and proximal parts of the esophagus is recommended. As the clinical symptoms of EoE do not correlate with the severity of the disease, a histopathological assessment is the basic tool in monitor the course of the disease and the effectiveness of the treatment process [9].

Since there is a strong association of EoE and atopic diseases, each patient suspected of EoE should undergo the allergological study. It includes laboratory tests such as peripheral blood eosinophilia, level of total lgE (clgE) and specific IgE serum test for food and inhalant allergens as well as blood cytokines levels [9, 11, 20]. In approx. $50 \%$ of patients with EoE, tests reveal peripheral eosinophilia and, therefore, a peripheral blood count with manual differential is recommended $[13,20]$. Peripheral eosinophilia may correlate with the degree of tissue infiltration by eosinophilic inflammation, but this test cannot be relied on as evidence of EoE and the marker of severity of the disease. An increased concentration of clgE may confirm atopy and allergies, however, normal clgE value does not exclude allergic diseases. There is no evidence
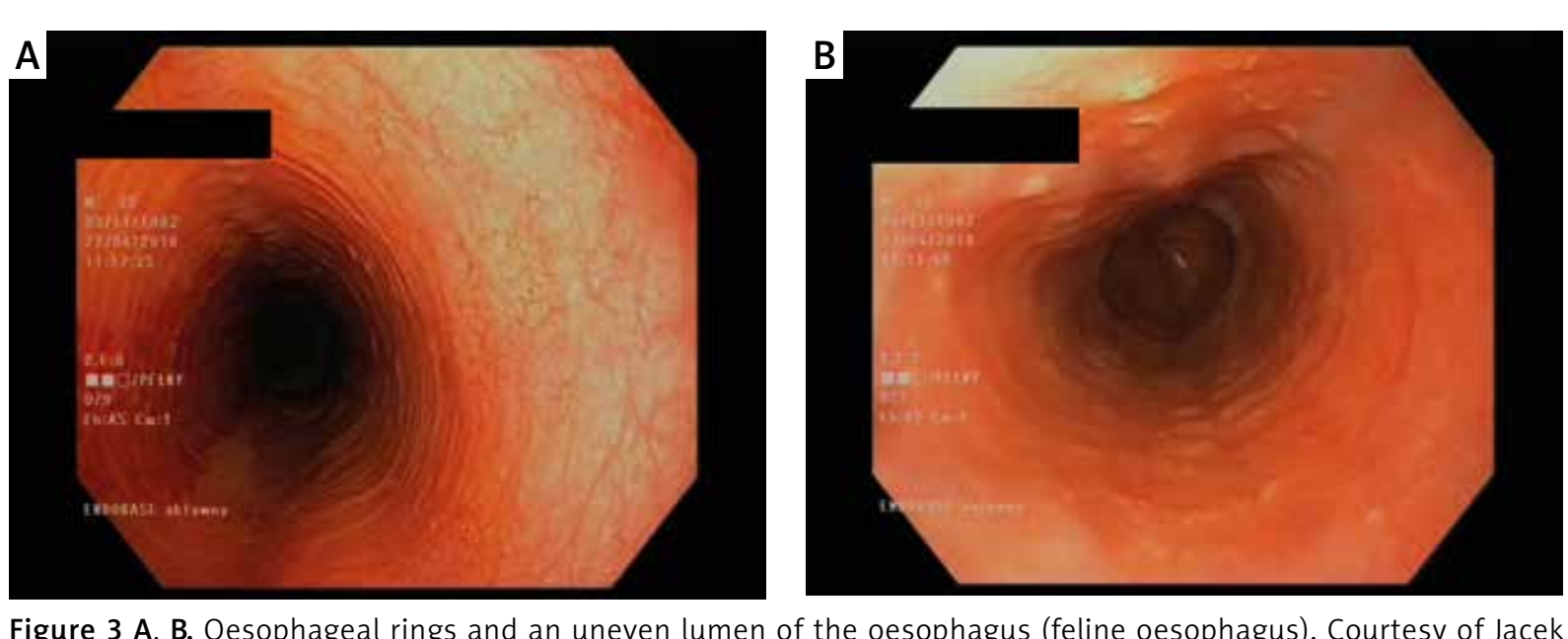

Figure 3 A, B. Oesophageal rings and an uneven lumen of the oesophagus (feline oesophagus). Courtesy of Jacek Muszyński, Department of Gastroenterology and Internal Medicine, Medical University of Warsaw

proving this test's value in assessment of the progression or remission of the disease [8]. Tests for IgE specific to food and inhalant allergens must be performed since there is a strong correlation between allergy and pathogenesis of EoE. It seems that assessing the expression of eotaxin-3 may be a useful tool in diagnosis and monitoring of EoE. However, it requires further research. In accordance with up-to-date knowledge, usefulness of measuring the levels of ECPs, tryptase, leukotrienes, MBPs, other cytokines or chemokines is questionable $[8,20]$.

Skin prick tests (PTS) are an easily available and fairly reliable diagnostic tool in testing for lgE-dependent allergic reactions. Positive results of inhalant allergy tests and a corresponding clinical picture are sufficient evidence of allergic reactions. Skin prick tests for food allergens are of low predictive value and introducing restrictive elimination diets by relying on positive PTS results alone is not recommended [8]. Atopy patch tests are used to confirm the late phase of the IgE-independent immunologic response. These tests are a well-recognised method in the diagnosis of atopic dermatitis and usually screen against food allergens, such as egg, milk, soy or wheat. It is, however, important to remember that these tests are

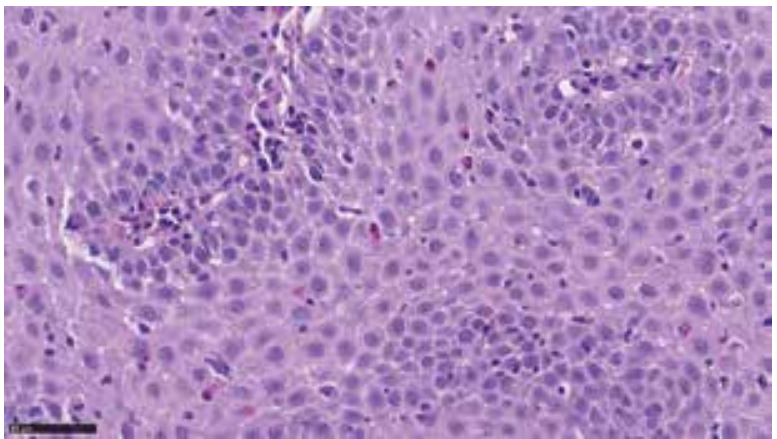

Figure 2. Eosinophilic infiltrations in oesophageal mucosa biopsy. Courtesy of Łukasz Michałowski, Department of Pathomorphology, Medical University of Warsaw 
not a standard method in diagnosis of allergies [8, 22]. Fast developing component diagnostic methods, based on molecular techniques, successfully applied in diagnostic testing for anaphylaxis and food allergies became also a promising tool in diagnosis of EoE [8].

In 2018, Warners et al. reported an interesting study regarding the use of esophageal pressure topography (EPT) tests in diagnosis for EoE. After a 4-week empiric diet, the authors injected food allergens into the esophageal mucous membrane of EoE and control group patients. Soy, wheat, egg and three food allergens based on patients' medical history were used in both groups. After $20 \mathrm{~min}$, endoscopic examinations with biopsies were performed and repeated after $24 \mathrm{~h}$. PTS and slgE for these specific allergens were also performed. Positive results of EPT tests were confirmed for 5 out of 8 patients after 20 min and for 2 patients after $24 \mathrm{~h}$. All the tests were negative in the control group. No correlation between the results of the skin prick tests/slgE tests and the results of the EPT tests was found. The authors suspect that allergic inflammation in EoE patients can only be present in the mucous membrane of the esophagus and, therefore, the results of slgE and skin prick tests for such patients are often negative [23]. In conclusion, a reliable tool in diagnosis of allergy in patients with EoE is still in the research phase [8].

Since the symptoms of EoE are not specific and gastrointestinal eosinophilia accompanies a number of conditions, differential diagnosis is required. Excluding other diseases with an increased number of epithelial eosinophils is one of the diagnostic criteria of EoE. These diseases include GERD, eosinophilic gastroenteritis, celiac disease, Crohn's disease, infection (including parasitic), pill esophagitis, hypereosinophilic syndrome, achalasia, hypersensitivity to drugs, pemphigoid, GvH disease and malignancies [10].

\section{Treatment}

The aims of the treatment of EoE are: clinical improvement (with the symptoms withdrawal and the quality of life improving) and histological improvement (to control the inflammation) [9].

In accordance with available research, the therapy of EoE is based on tree pillars: pharmacological treatment, elimination diets and endoscopic esophageal dilation $[9,11,20]$. The first two are expected to reduce the inflammation, while the purpose of the third is to eliminate the effects of post-inflammatory fibrosis (an esophageal stricture).

\section{Elimination diets}

\section{Dietary treatment}

Allergens introduced with food may induce EoE, which will resolve once an appropriate elimination diet is implemented. Three dietary strategies are currently in use: elemental diet (ED), allergy testing-based food elimi- nation diet (ATBD) and empiric six-food group elimination diet (SFED) (or, optionally, with the elimination of 4 or 2 allergens).

\section{Elemental diet}

An elemental diet is a specific, well-balanced, allergen-free diet. The effectiveness of this diet is very high and reaches $>90 \%$. A 6-week course of this treatment usually results in the withdrawal of the clinical and histological symptoms. The disadvantage of this treatment is unpleasant taste and smell of the products [9, 13, 20, 24].

\section{Allergy testing-based food elimination diet}

This diet eliminates certain foods relying on positive results of skin prick tests and slgE tests. Its effectiveness rate is approx. $45.5 \%$. Since skin prick tests and slgE tests are of low predictive value in the diagnostics of food allergies, this way of treatment is often ineffective. However, if such tests are accompanied by keeping a diary and self-monitoring, the results are better.

\section{Empiric six-food group elimination diet}

This diet empirically eliminates food allergens such as soy beans, wheat, milk, egg, fish and seafood, and peanuts. It results in clinical improvement in $75 \%$ of patients $[9,11,13,20,24]$. The diet must be kept for 6 weeks before endoscopic examination looking for regression of inflammation can be performed. If the diet has resulted in clinical improvement, the eliminated foods are reintroduced watchfully, followed by endoscopic examinations for histological assessment. Any food proven to promote the development of EoE must be eliminated from the patient's diet permanently.

A limitation of this treatment is a large number of endoscopic examinations, which is rarely accepted by the patients. Dietary treatment should be provided by a team of specialists, including a gastroenterologist, an allergist and a dietitian $[9,11,13,20,24]$.

\section{Pharmacological treatment (Table 1) \\ Proton pump inhibitors (PPIs)}

Approximately $50 \%$ of patients meeting the criteria for EoE improve on proton pump inhibitors (PPI) monotherapy. Previously, an outcome of an 8-week high-dose PPI trial was considered a therapeutic and diagnostic criterion of proton pump inhibitor responsive esophageal eosinophilia (PPI-REE). The name PPI-REE has now been replaced with $\mathrm{PPI}$-responsive EoE [9].

\section{Topical glucocorticosteroids}

Many studies have proven the effectiveness of this group of medications in the treatment of EoE. Budesonide and fluticasone are commonly in use. Fluticasone should be administered using metered dose 
Table 1. Pharmacological treatment in EoE

\begin{tabular}{|c|c|c|c|}
\hline Drug & Children & Adults & Maintenance \\
\hline \multicolumn{4}{|l|}{ Proton pump inhibitor: } \\
\hline \multirow[t]{2}{*}{ Omeprazole or esomeprazole } & 10-20 kg - 10 mg twice daily & \multirow[t]{2}{*}{40 mg twice daily } & \multirow{2}{*}{$\begin{array}{c}\text { After } 8 \text { weeks, minimal } \\
\text { effective dose }\end{array}$} \\
\hline & $>20 \mathrm{~kg}-20 \mathrm{~kg} / \mathrm{mg}$ twice daily & & \\
\hline \multicolumn{4}{|l|}{ Topical corticosteroids: } \\
\hline Fluticasone & 88-440 $\mu$ g twice daily & 440-880 $\mu$ g twice daily & \multirow[t]{2}{*}{ Minimal effective dose } \\
\hline Budesonide & 0.5 mg twice daily & $1 \mathrm{mg}$ twice daily & \\
\hline \multicolumn{4}{|l|}{ Systemic corticosteroids: } \\
\hline Prednisone & & $1-2 \mathrm{mg} / \mathrm{kg} /$ day for $10-14$ days & \\
\hline
\end{tabular}

inhalers in doses of $440-880 \mu \mathrm{g}$ daily. A patient must apply the medication into the oral cavity and then swallow. Budesonide is administered in doses of 0.5-0.6 mg daily. Usually patients are advised to drink the solution used for nebulisations. It is recommended that a sweetener (sucralose or honey) be added to the suspension to increase its viscosity [13]. The patient must not eat or drink for 30 min after swallowing the medication. The effectiveness of the therapy should be verified after 6-8 weeks. However, many patients require long-term therapy. It is believed that the smallest effective dose of topical glucocorticosteroids should be used in the therapy $[9,13,20]$.

A great turning point in the treatment of EoE is the registration, by FDA and EMA, of a new form of budesonide, namely tablets that dissolve in the mouth. Patients find this form of the medication easy to use [25].

Systemic glucocorticosteroids are highly effective in EoE therapy. However, given their adverse effects, they should only be used in severe cases of EoE and over short-term courses.

The preferred choice is prednisolone in a dose of 1-2 mg for each kilogram of the patient's body weight, for 10-14 days [9].

\section{Mechanical esophagus dilation}

This method is used only in patients in whom esophageal stricture has occurred despite pharmacological treatment. This method proves effective for $75 \%$ of patients. The procedure should be performed in adequately experienced gastroenterological centres only $[9,11,13$, 20, 24].

Research into new methods for EoE treatment continues. Medications of biological origin offer great hope. However, clinical trials with monoclonal antibodies (anti-IL5 (reslizumab), anti-TNF (infliximab), anti-IgE (omalizumab)) have not proved these medications to be effective. Clinical trials continue with the following monoclonal antibodies: anti-IL-13, anti-CRTH2, anti-IL-4 and anti-TGF- $\beta$ ) [11].

\section{Conclusions}

The increasing incidence of allergic diseases causes new challenges in the field of medicine. Gastrointestinal symptoms have been known to reduce quality of life significantly in all age groups. Eosinophilic esophagitis strongly correlating with atopic and allergic disorders comes to attention of allergologists and gastroenterologists. Since gastrointestinal endoscopy and mucosal biopsy are main tools of diagnosis and assessment of effectiveness of treatment, less invasive procedures must be invented to avoid complications and improve patients' comfort and compliance. Research continues, but little is known about cellular pathomechanisms of the disease. Therefore specific treatment, although increasing effectiveness requires further improvement and additional evidence, is needed to establish effective treatment options.

\section{Conflict of interest}

The authors declare no conflict of interest.

\section{References}

1. Landres RT, Kuster GG, Stum WB. Eosinophilic esophagitis in a patient with vigorous achalasia. Gastroenterology 1978; 74: 1298-301.

2. Picus D, Frank PH. Eosinophilic esophagitis. AJR Am J Roenthenol 1981; 136: 1001-3.

3. Attwood SEA, Smyrk TC, Demeester TR. Esophageal asthma an episodic dysphagia with eosinophilic infiltrates. Gut 1989; 30: A1493.

4. Attwood SEA, Smyrk TC, Demeester TR, Jones JB. Esophageal eosinophilia with dysphagia. A distinct clinical pathologic syndrome. Dig Dis Sci 1993; 38: 109-16.

5. Straumann A, Spichtin HP, Bernoulli R, et al. Idiopathic eosinophilic esophagitis: a frequently overlooked disease with typical clinical aspects and discreate endoscopic findings. Schwitz Med Wochednschr 1994; 124: 1419-29.

6. Atwood SEA, Furuta GT. Eosinophilic esophagitis: historical perspective on an evolving disease. Gastroenterol Clin North An 2014; 43: 185-99.

7. Kelly KJ, Lazemby AJ, Rowe PC, et al. Eosinophylic esophagitis attributed to gastroesophageal reflux: improvement with an amino acid-based formula. Gastroenterology 1995; 109: 1503-12. 
8. Anyane-Yeboa A, Wang W, Kavitt RT. The role of allergy testing in eosinophilic esophagitis. Gastroenterol Hepatol 2018; 14: 463-9.

9. Lucendo AJ, Molina-Infante J, Arias A, et al. Guideline on eosinophilic esophagitis: evidence-based statements and recommendations for diagnosis and management in children and adults. United Eur Gastroenterol I 2017; 5: 335-58.

10. Kim HP, Dellon ES. An evolving approach to the diagnosis of eosinophilic esophagitis. Gastroenterol Hepatol 2018; 14: 358-66.

11. Gomez Torrijos E, Gonzalez-Mendiola R, Alvarado M, et al. Eosinophilic esophagitis: review and update. Front Med 2018; 5: 247.

12. Sato H, Honma T, Nozawa Y, et al. Eosinophilic esophagitis in Japanese patients: a mild and slow-progressing disorder. PLoS One 2018; 13: e0206621.

13. Carr S, Chan ES, Watson W. Eosinophilic esophagitis. Allergy Asthma Clin Immunol 2018; 14 (Suppl 2): 58.

14. Cianferoni A, Spergel JM. From genetics to treatment of eosinophilic esophagitis. Curr Opin Allergy Clin Immunol 2015; 15: 417-25.

15. Avila-Castellano R, Garcia-Lozano JR, Cimbillek S, et al. Genetic variations in the TLR3 locus are associated with eosinophilic esophagitis. United Eur Gastroenterol J 2018; 6: 349-57.

16. Cingi C, Gevaert P, Mosges R, et al. Multi-morbidities of allergic rhinitis in adults: European Academy of Allergy and Clinical Immunology task force report. Clin Trans Allergol 2017; 7: 17.

17. Aceves SS. Food allergy testing in eosinophilic esophagitis: what the gastroenterologist needs to know. Clin Gastroenterol Hepatol 2014; 12: 1216-23.

18. Gołąb J, Jakóbisiak M, Lasek W, Stokłosa T. Immunologia. Wydawnictwo Naukowe PWN, Warsaw 2018.

19. Hill DA, Spergel JM. The immunologic mechanisms of eosinophilic esophagitis. Curr Allergy Asthma Rep 2016; 16: 9.

20.Simon D, Straumann A, Schoepfer AM, et al. Current concepts in eosinophilic esophagitis. Allergo J Int 2017; 26: 258 66.

21. Aiolfi A, Ferrari D, Riva CG, et al. Esophageal foreign bodies in adults: systemic review of the literature. Scand J Gastroenterol 2018; 53: 1171-8.

22. Soylu A, Altintes A, Cakmak S. The coexistence of eosinophilic esophagitis with allergic rhinitis. Eur Rev Med Pharmacol Sci 2016; 20: 2315-23.

23. Warners MJ, Terreehorst I, van den Wijngaard MR, et al. Abnormal responses to local esophageal food injection in adult patients with eosinophilic esophagitis. Gastroenterology 2018; 154: 57-60.

24. Reed CC, Safta AM, Quasem S, e. al. Combined and alternating topical steroids and food elimination diet for the treatment of eosinophilic esophagitis. Dig Dis Sci 2018; 63: 2381-8.

25. Dellon ES, Katzka DA, Collins MH, et al. Budesonide oral suspension improves symptomatic, endoscopic, and histologic parameters compared with placebo in patients with eosinophilic esophagitis. Gastroenterology 2017; 152: 776-86. 